\title{
Comparison between Dirichlet-Robin and Neumann-Robin Interface Conditions in CHT Problems
}

\author{
Rocco Moretti, Marc Errera \\ DAAA, ONERA, Université Paris Saclay \\ 29 Avenue de la Division Leclerc, 92320 Châtillon, France \\ rocco.moretti@onera.fr; marc.errera@onera.fr
}

\begin{abstract}
This paper presents and compares the stability and the performance of two different boundary conditions in steady conjugate heat transfer (CHT) problems. The Dirichlet-Robin interface and the Neumann-Robin interface condition have been implemented. The choice between the two interface conditions depends on the numerical Biot number that is a local representation of the thermal fluid-structure interaction and plays a key role in the stability of the coupling process. The coupling coefficient of the Robin condition is determined by the transition of the amplification factor derived from a normal mode stability analysis based on the Godunov-Ryabenkii theory. A study of an academic 2D test case illustrates that, although the Dirichlet-Robin interface condition is optimal for low and moderate thermal fluid-structure interaction, the Neumann-Robin condition is a relevant choice at higher thermal fluid-structure interaction.
\end{abstract}

Keywords: CHT, Neumann, Dirichlet, Robin, Stability, Optimal Coefficient, Biot Number, Boundary Condition.

\section{Introduction}

The aim of CHT problems is to solve the thermal interaction between a body and a fluid flowing over or through it. This numerical process takes into account the fluid convection and the solid conduction simultaneously. Perelman was the first to work on it [1]. In the literature, there are two ways to solve CHT problems or fluid-structure interaction problems in general. The first one is the monolithic method in which the equations are solved simultaneously in a single solver [2], the second one is the partitioned method in which every domain is solved separately updating the boundary conditions at the common interface [3]. The monolithic method presents good stability properties but a specific solver capable of solving all physics simultaneously is required. This needs a unique time step for both solvers resulting in a large computational cost. On the other hand, partitioned methods allow us to use an appropriate solver for each physical problem but they present serious stability issues.

Many studies were carried out in the last decades to identify stabilizing procedures or techniques for partitioned methods. Particularly in CHT, the effects of the interface conditions are often studied using a normal mode analysis. Errera and Chemin have proposed an analytical expression of an optimal Robin coefficient capable of stabilizing most of the CHT problems [4]. This expression has been derived from a normal mode analysis based on the Godunov-Ryabenkii theory [5]. This optimal coefficient has been tested in various works [6][7][8] and it has been used also in the present paper.

\section{Interface Treatment}

The aim of this work is to show the stability properties of the most common interface treatments in CHT problems:

- The Dirichlet-Robin procedure: the Dirichlet condition on the fluid side and the Robin condition on the solid side.

- The Neumann-Robin procedure: the Neumann condition on the fluid side and the Robin condition on the solid one.

\subsection{Stability Analysis}

A normal mode stability analysis applied to a discrete CHT model problem with a Robin condition on both sides of the coupled interface (fluid and solid) leads to the following expression of the temporal amplification factor $g$ [4]: 


$$
g\left(\alpha_{f}, \alpha_{s}\right)=\frac{\left(\alpha_{f}+\alpha_{s}\right) K_{f}}{\left(K_{f}+\alpha_{s}\right)\left(\alpha_{f}+K_{s}\right)} \kappa_{f}+\frac{\left(K_{s}-\alpha_{s}\right)\left(K_{f}-\alpha_{f}\right)}{\left(K_{f}+\alpha_{s}\right)\left(\alpha_{f}+K_{s}\right)}
$$

where $K_{f}$ and $K_{s}$ are the fluid and solid conductances respectively $\left[\mathrm{Wm}^{-2} \mathrm{~K}^{-1}\right], \alpha_{f}$ and $\alpha_{s}$ are the coupling coefficients for the solid and fluid Robin boundary conditions respectively and $\kappa_{f}$ is the spatial amplification factor:

$$
\kappa_{f}=\kappa_{f}\left(D_{f}, z\right)=1+\frac{z-1}{2 D_{f} z}-\sqrt{\left(1+\frac{z-1}{2 D_{f} z}\right)^{2}-1}
$$

where $\mathrm{D}_{\mathrm{f}}$ is the Fourier number that is directly linked to the fluid thermal diffusivity $a_{f}$, the coupling time step $\Delta t_{c}$ and the fluid mesh size near the coupled wall $\Delta y_{f}$ :

$$
D_{f}=\frac{a_{f} \Delta t_{c}}{\Delta y_{f}^{2}}
$$

\subsection{The Optimal Dirichlet-Robin Procedure}

This procedure consists of imposing the temperature coming from the solid solver on the fluid side $\left(\alpha_{s}=\infty\right)$ and the Robin condition on the solid side, i.e. a linear combination of the temperature and the heat flux calculated by the fluid solver as shown in Eq. (2):

$$
\hat{q}_{s}+\alpha_{f} \widehat{T}_{s}=-q_{f}+\alpha_{f} T_{f}
$$

where $q$ is the normal heat flux and $T$ is the temperature. The superimposed hat symbol ( $\left.{ }^{\wedge}\right)$ denotes the sought values.

As the name of the subsection indicates, we use the optimal procedure for the Dirichlet-Robin interface condition, that is to say, we impose the optimal value expressed by:

$$
\alpha_{f}^{(o p t)}=\frac{K_{f}}{2}\left(1-\bar{D}_{f}\right)
$$

where the $\overline{\mathrm{D}}_{\mathrm{f}}$ is a normalized Fourier number:

$$
\bar{D}_{f}=\frac{D_{f}}{1+D_{f}+\sqrt{1+2 D_{f}}}
$$

For this value of the coupling coefficient the minimum of the temporal amplification factor is attained (see Fig. 1), that is to say the fastest convergence rate is obtained theoretically. Figure 1 introduces the numerical Biot number $\mathrm{Bi}_{v}$, a dimensionless number that measures the intensity of the thermal fluid-structure interaction. This number is the y-intercept of the amplification factor curve. For high values of the numerical Biot number, the temporal amplification factor takes values greater than unity (stability limit according to the Godunov-Ryabenkii theory) resulting in unstable CHT simulations. 


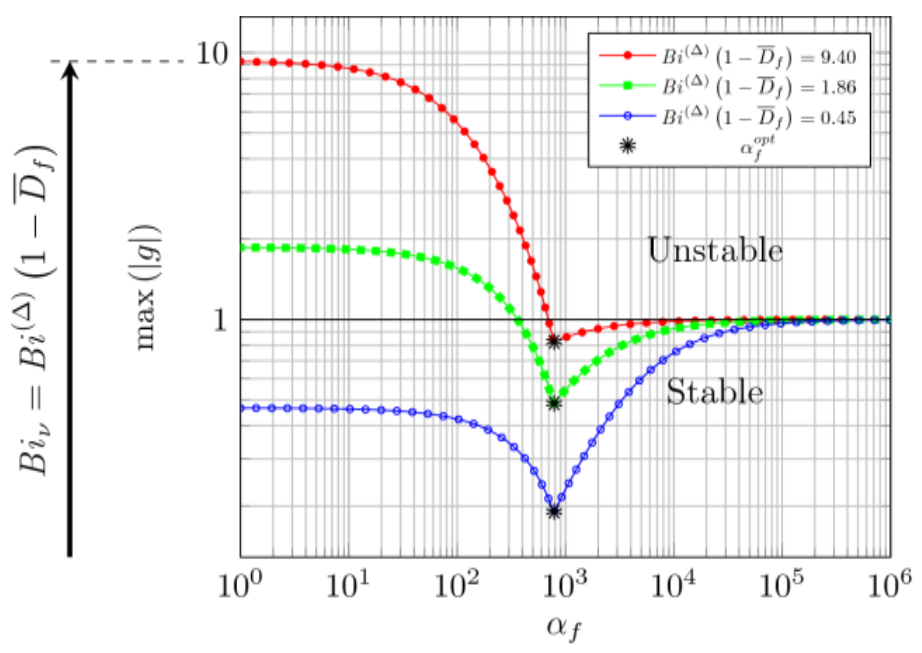

Fig. 1: Temporal amplification factor $(\max |g|)$ and optimal coefficient for three different intensity values of the fluid-structure interaction $B i_{v}$ in the case of Dirichlet-Robin interface procedure [9].

\subsection{The Optimal Neumann-Robin Procedure}

This procedure is complementary to the optimal Dirichlet-Robin procedure because a normal heat flux is imposed on the fluid side $\left(\alpha_{s}=0\right.$.) in place of the temperature.

Using these new boundary conditions the expression of the temporal amplification factor changes and consequently a new optimal coefficient is obtained:

$$
\alpha_{f}^{(o p t)}=\frac{2 K_{s} K_{f}}{2 K_{s}-K_{f}\left(1+\bar{D}_{f}\right)}=\frac{2 K_{f}}{2-B i^{(\Delta)}\left(1+\bar{D}_{f}\right)}
$$

where $B i^{(4)}$ is the mesh Biot number, i.e. the ratio between the fluid and the solid thermal conductances.

Figure 2 shows the temporal amplification as a function of the coupling coefficient for Neumann-Robin interface procedure. If the thermal interaction is weak (small values of the Biot number) the right part of the curve is in the unstable zone and exhibits an optimal coefficient. For very strong thermal interaction no optimal coefficient exists, so the Neumann-Dirichlet interface procedure is the best choice.

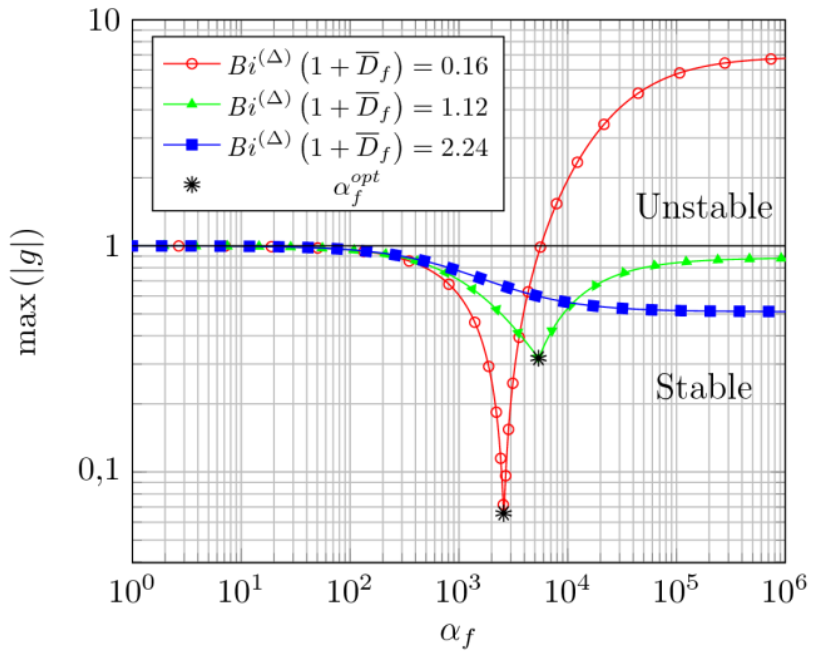

Fig. 2: Temporal amplification factor $(\max |g|)$ and optimal coefficient for three different intensity values of the fluid-structure interaction in the case of Neumann-Robin interface procedure [9]. 


\section{CHT Computations \\ 3.1. CHT Test Case}

In this section a comparison between the two interface procedures is presented. Results will vary depending on the intensity of the thermal interaction (estimated by $B i_{v}$ ). This number can be changed easily by modifying the solid conductance $K_{s}$. The test case chosen in this paper is a CHT problem composed by convective heat transfer over, and conduction within, a flat plate.

\subsection{Results}

(a)

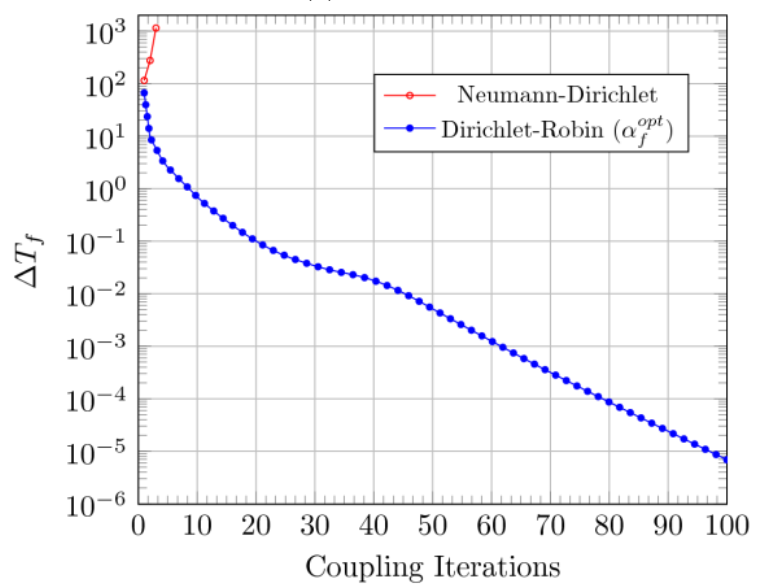

(b)

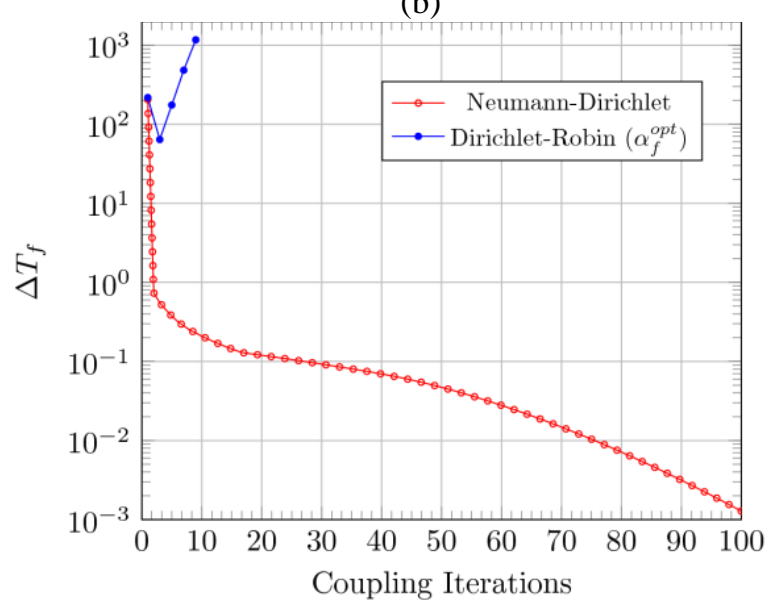

Fig. 3: Convergence vs coupling iterations. (a) Weak thermal interaction $\left(\lambda_{\mathrm{s}}=10.0 \mathrm{Wm}^{-1} \mathrm{~K}^{-1}, B i_{v}=0.47\right)$.

(b) Strong thermal interaction $\left(\lambda_{\mathrm{s}}=0.01 \mathrm{Wm}^{-1} \mathrm{~K}^{-1}, B i_{v}=462.5\right)$ [9].

Figures $3 \mathrm{a}$ and $3 \mathrm{~b}$ show the convergence history of the interface temperature (infinity norm). If the thermal fluidstructure interaction is very weak $\left(B i_{v}<1\right.$.), the CHT problem results unstable using the Neumann-Dirichlet interface procedure but it is stable and fast converging when the Dirichlet-Robin procedure is applied (Fig. 3a). However, in the case of strong thermal interaction $\left(B i_{v} \gg>1\right.$.), the common procedure used in the literature, i.e. the Dirichlet-Robin procedure, results in a rapid divergence of the CHT simulation (Fig. 3b). In this case, the Neumann-Dirichlet procedure assures the convergence of the simulation.

\section{Conclusion}

We have shortly presented in this paper the main stability properties of two different interface procedures, the Dirichlet-Robin and the Neumann-Dirichlet procedures. Some important numbers, i.e. the Fourier number and the numerical Biot number, have been introduced. These numbers include media thermal properties, as you can find in the literature, but also some numerical parameters able to adapt the exchanged quantities to the numerical problem (mesh size, coupling time period, etc...). The numerical number can be employed to identify the most suitable interface procedure. In conclusion we have shown that the choice of the boundary conditions can affect significantly the stability of the coupling problem as well as the best interface procedure depends on the strength of the thermal F-S interaction. Therefore, we may note that the Dirichlet-Robin is the optimal interface condition for low and moderate thermal fluid-structure interaction, whereas the Neumann-Robin interface condition is a relevant choice at higher thermal fluid-structure interaction. 


\section{References}

[1] T. L. Perelman, "On conjugated problems of heat transfer," Int. J. Heat Mass Transfer, vol. 3, no. 4, pp. 293-303, 1961.

[2] A. Matalip, N. Wansophark, P. Dechaumphai, "Combined streamline Upwind Petrov Galerkin Method and segregated finite element algorithm for conjugate heat transfer problems," J. Mech. Sci. Technol., vol. 20, no. 10, pp. 1741-1752, 2006.

[3] C. A. Felippa, K. C. Park, "Staggered transient analysis procedures for coupled dynamic systems: formulation," Comput. Methods Appl. Mech. Eng., vol. 24, no. 1, pp. 61-111, 1980.

[4] M. P. Errera, S. Chemin, "Optimal solutions of numerical interface conditions in fluid-structure thermal analysis," J. Comput. Phys., vol. 245, supplement C, pp. 431-455, 2013.

[5] S. K. Godunov, V. S. Ryabenkii, The theory of difference schemes: an introduction. Amsterdam, North-Holland, 1964.

[6] M. P. Errera, G. Turpin, "Temporal multiscale strategies for conjugate heat transfer problems," J. Coupled Syst. Multiscale Dyn., vol. 1, no. 1, pp. 89-98, 2013.

[7] M. P. Errera, F. Duchaine, "Comparative study of coupling coefficients in Dirichlet-Robin procedure for fluidstructure aerothermal simulations," J. Comput. Phys., vol. 312, supplement C, pp. 218-234, 2016.

[8] R. El Khoury, M. P. Errera, K. El Khoury, M. Nemer, "Efficiency of coupling schemes for the treatment of steady state fluid-structure thermal interactions," Int. J. Therm. Sci., vol. 115, pp. 225-235, 2017.

[9] R. Moretti, M. P. Errera, V. Couaillier, F. Feyel, "Stability, convergence and optimization of interface treatments in weak and strong thermal fluid-structure interaction," Int. J. Therm. Sci., vol. 126, pp. 23-37, 2018. 